\title{
Validation of Ray Tracing Code Refraction Effects
}

\author{
Stephanie L. Heath ${ }^{*}$ and Gerry L. McAninch ${ }^{\dagger}$ \\ NASA Langley Research Center, Hampton, Virginia 23681-2199 \\ Charles D. Smith \\ Lockheed Martin Information Technology, Hampton, Virginia 23681-2199 \\ David A. Conner ${ }^{\S}$ \\ US Army, AFDD-JRPO, AMRDEC, RDECOM, Hampton, Virginia 23681-2199
}

\begin{abstract}
NASA's current predictive capabilities using the ray tracing program (RTP) are validated using helicopter noise data taken at Eglin Air Force Base in 2007. By including refractive propagation effects due to wind and temperature, the ray tracing code is able to explain large variations in the data observed during the flight test.
\end{abstract}

\section{Introduction}

$\mathrm{N}$ ASA has developed a ray tracing aircraft acoustics propagation program, $\mathrm{RTP}^{1}$, based on geometric acoustic theory ${ }^{2,3,4}$, in order to account for refractive effects due to wind and temperature gradients. Theoretical studies using RTP have demonstrated that even slight atmospheric changes can significantly impact resulting sound pressure levels on the ground at long distances ${ }^{5}$. Now, in order to ensure correct physical predictions, NASA will validate RTP against actual flight data. Level flight data from a Mil MI-8 helicopter acquired on September 8 and 9, 2007, during the 2007 Flight Test at Eglin Air Force Base, will be used for validation.

\section{Test Site Conditions}

Test range C-72 at Eglin Air Force Base in Florida was used for this experiment. The test range spans a longitudinal distance of five miles, and, with the exception of a couple of sharp valleys, the terrain is flat. An aerial view of the test site is shown in Figure 1.

Testing was performed in the early morning hours. Table 1 below shows the runs used to validate RTP. All cases are steady level flights at an altitude of 300 feet and a speed of 150 knots, with the vehicle moving toward the microphone. While it does not greatly affect the results it should be noted that, due to safety concerns, the helicopter did not fly directly over the microphone, but had an offset of approximately 300 feet. The primary difference between these cases is the relative direction between the wind and the flight path directions. Two flight path headings were used for this study, and are shown on Figure 1. Flight path headings, by convention, are designated as zero degrees when aligned with north and incremented clockwise to three hundred sixty degrees.

Table 1 also indicates the corresponding atmospheric conditions used in the propagation predictions. The atmospheric conditions were obtained from weather balloon data.

Table 1. Summary of runs used for RTP validation.

\begin{tabular}{|l|l|l|l|l|l|l|}
\hline $\begin{array}{c}\text { Case } \\
\text { Number }\end{array}$ & Run Date & Run No & $\begin{array}{c}\text { Run } \\
\text { Start } \\
\text { Time }\end{array}$ & $\begin{array}{c}\text { Flight Path } \\
\text { Heading }\end{array}$ & $\begin{array}{c}\text { Atmospheric } \\
\text { Condition } \\
\text { (see Figures 2 } \\
\text { and 3) }\end{array}$ & $\begin{array}{c}\text { Atmospheric Balloon } \\
\text { Data } \\
\text { (reference time) }\end{array}$ \\
\hline 1 & $9 / 8 / 2007$ & 501702 & $7: 11 \mathrm{AM}$ & $100 \mathrm{deg}$ & 1 & $7: 06-7: 35 \mathrm{AM}$ \\
\hline 2 & $9 / 8 / 2007$ & 501704 & $7: 45 \mathrm{AM}$ & $20 \mathrm{deg}$ & 2 & $7: 35-7: 54 \mathrm{am}$ \\
\hline 3 & $9 / 9 / 2007$ & 502711 & $7: 00 \mathrm{AM}$ & $100 \mathrm{deg}$ & 3 & $6: 42-7: 16 \mathrm{AM}$ \\
\hline 4 & $9 / 9 / 2007$ & 502713 & $7: 38 \mathrm{AM}$ & $20 \mathrm{deg}$ & 4 & $7: 38-8: 14 \mathrm{AM}$ \\
\hline
\end{tabular}

* Aeronautical Engineer, Aeroacoustics Branch, MS 461 NASA LaRC, Member AIAA.

${ }^{\dagger}$ Sr. Aeronautical Engineer, Structural Acoustics Branch, MS 463 NASA LaRC.

*Aeronautical Engineer, Lockheed Martin Information Technology, MS 461 NASA LaRC.

$\S$ Aeronautical Engineer, US Army, MS 461 NASA LaRC. 


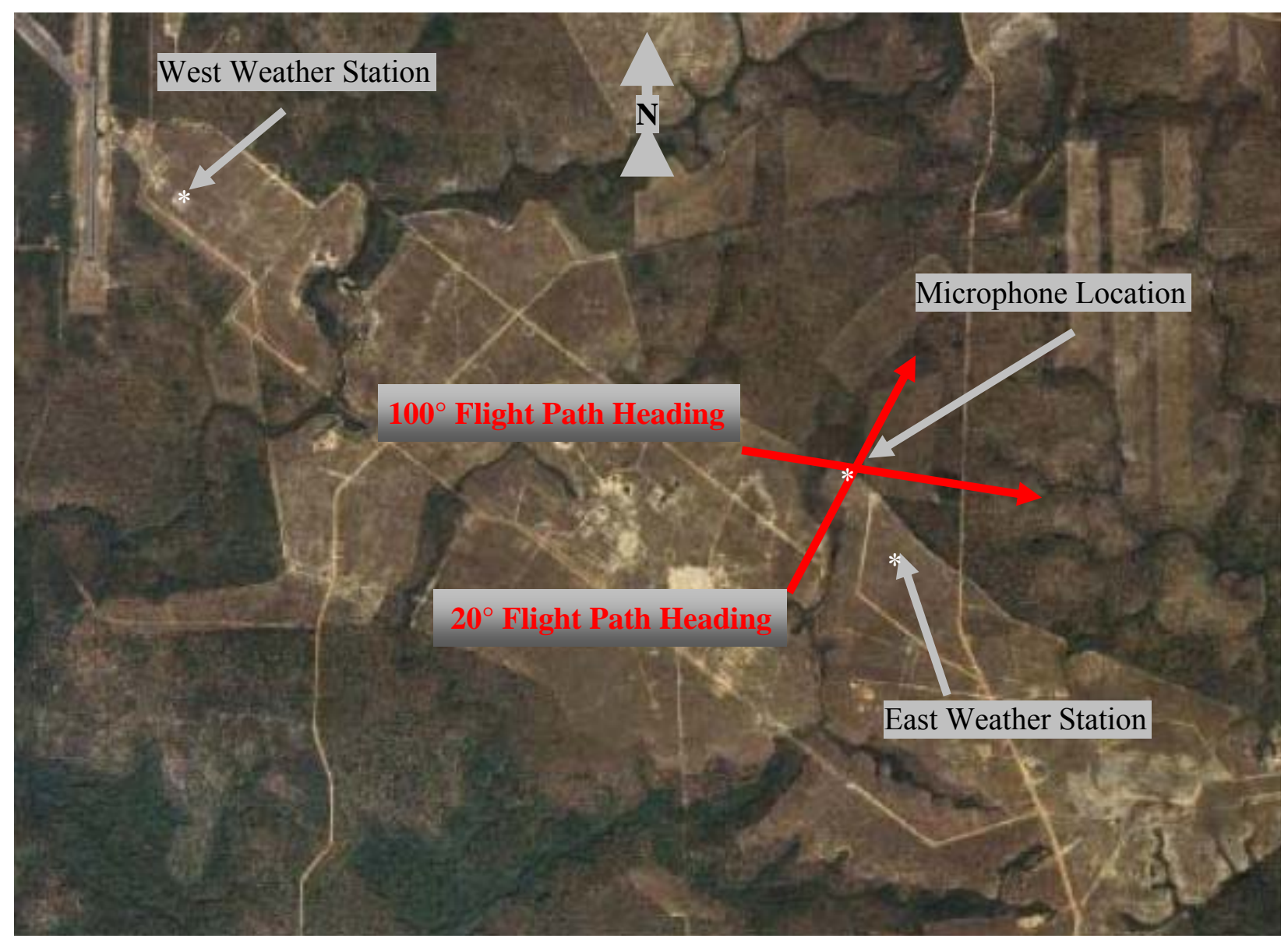

Figure 1. Aerial view of Eglin Air Force Base C-72 Test Site.

\section{Meteorological Data}

Two weather balloons were located at the test site, one at the east and one at the west side of the test site, as seen in Figure 1. A cursory comparison shows similar profiles between the west and the east weather balloons. This indicates that the atmosphere remains stratified over the test site, and that data from one of the balloons is sufficient to characterize the test site. Since the east weather balloon was disabled for safety reasons during our flights, only data from the west balloon are available. The weather balloon obtained profiles of temperature, relative humidity, wind speed, and wind direction up to an altitude of 3000 feet. Data were gathered continuously during the morning hours, with complete profiles obtained about every 20 minutes.

The temperature profiles for the four atmospheric conditions referenced in Table 1 correspond to the four flight cases. The atmospheric profiles are plotted in Figures 2 (temperature) and 3 (wind). The temperature profiles for atmospheric conditions 1 and 2 (Figure $2 \mathrm{~b}$ ) show a slight positive lapse rate (temperature decreasing with increasing altitude) between the ground and the source height of 300 feet. Atmospheric conditions 3 and 4 shown in Figure $2 \mathrm{~d}$ are inverted (temperature increasing with increasing altitude) and almost constant, respectively.

The winds at the test site are shown in Figure 3. By convention, the wind direction is labeled as northerly if the wind is blowing from the north to the south, and is designated as zero degrees. The wind conditions shown are relatively mild and generally blowing from the east at approximately 100 degrees. The wind speeds at the source height of $300 \mathrm{feet}$ are approximately $20 \mathrm{ft} / \mathrm{sec}$ for all flights. 


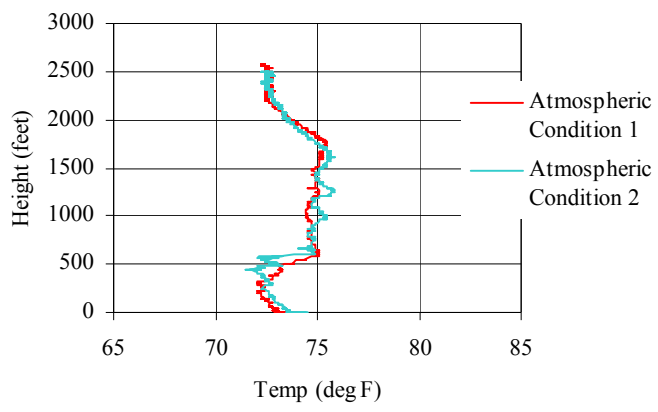

(a). Temperature profiles for altitudes up to 3000 feet for conditions 1 and 2 .

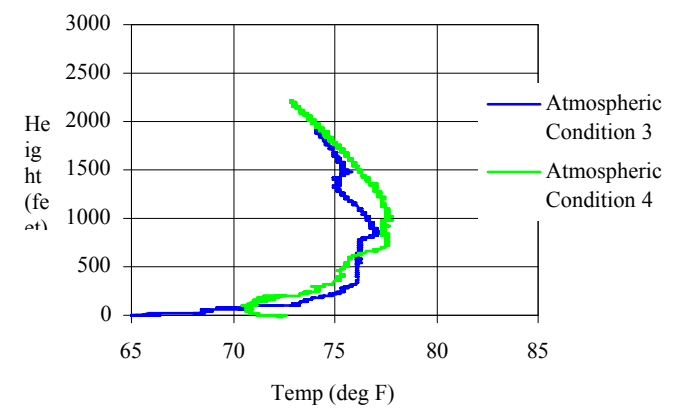

(c). Temperature profiles for altitudes up to 3000 feet for conditions 3 and 4 .

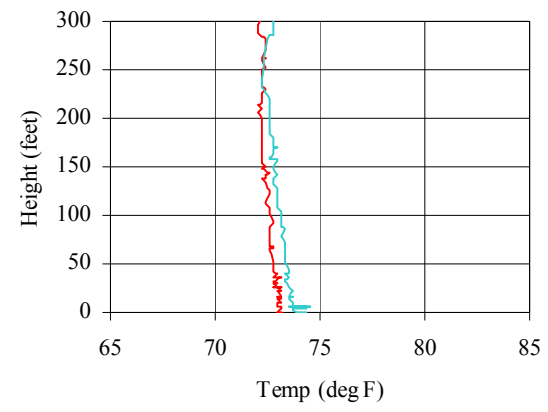

(b). Temperature profiles for altitudes up to 300 feet for conditions 1 and 2.

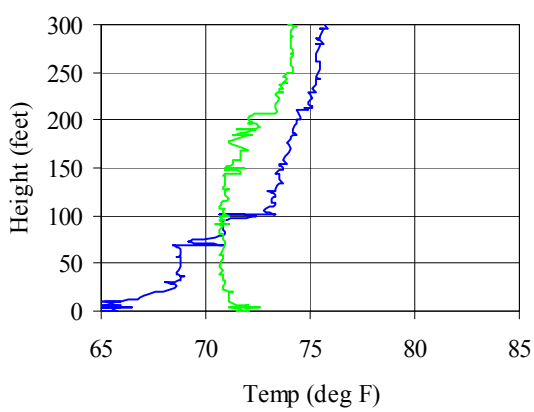

(d). Temperature profiles for altitudes up to 300 feet for conditions 3 and 4 .

Figure 2. Temperature profiles for the four atmospheric conditions.

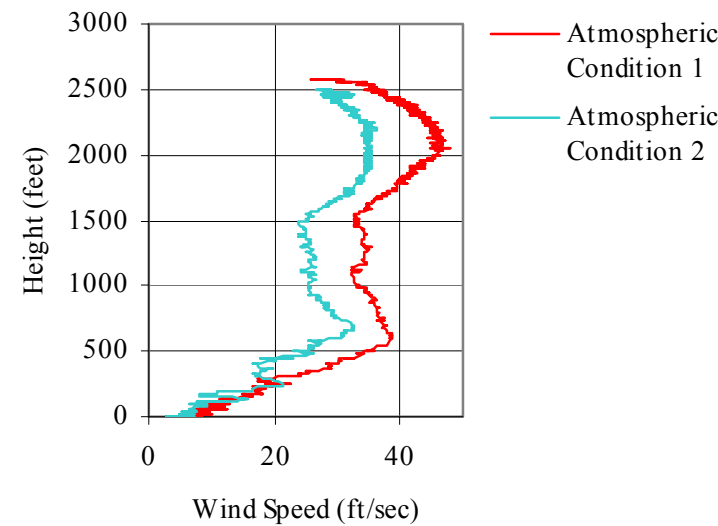

(a). Wind speed profiles for altitudes up to 3000 feet for conditions 1 and 2.

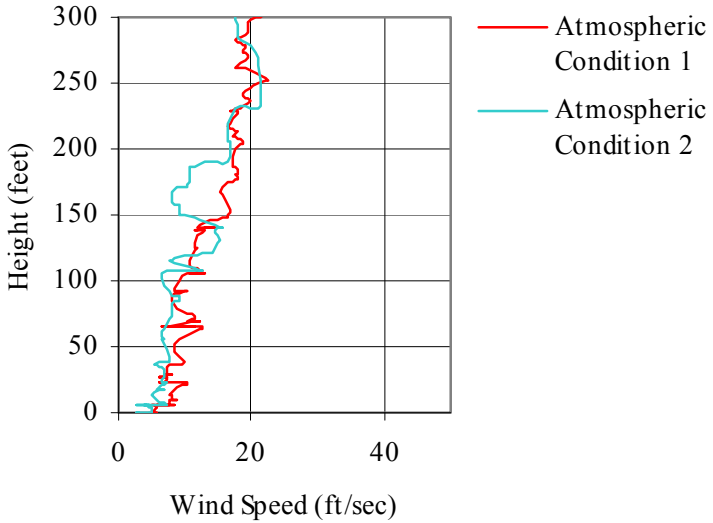

(b). Wind speed profiles for altitudes up to 300 feet for conditions 1 and 2 . 


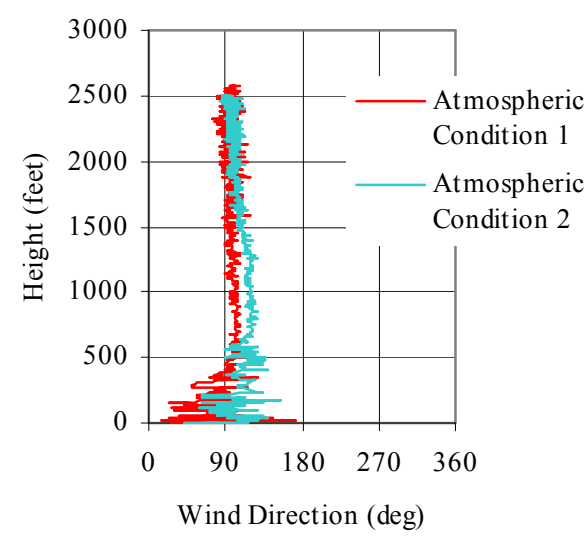

(c). Wind directions for altitudes up to 3000 feet for conditions 1 and 2.

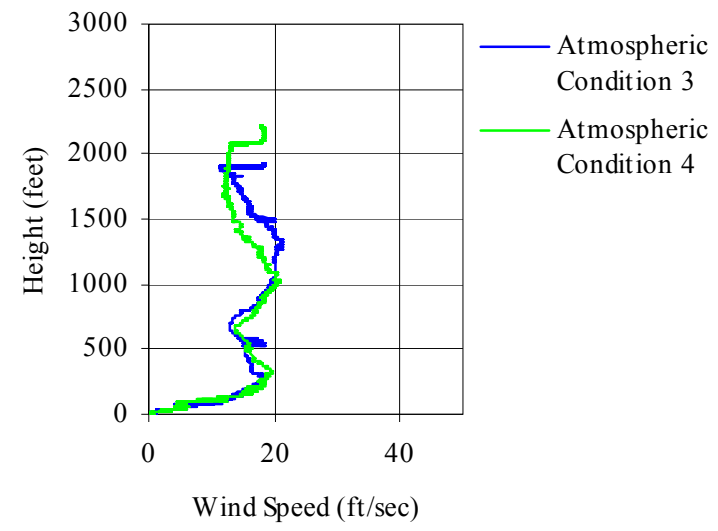

(e). Wind speed profiles for altitudes up to 3000 feet for conditions 3 and 4 .

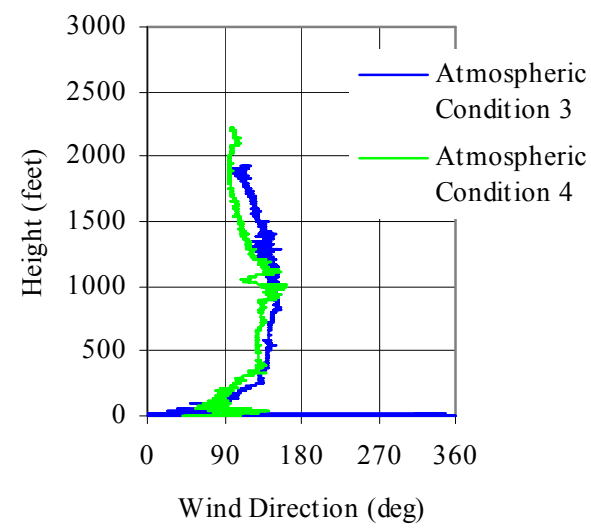

(g). Wind direction for altitudes up to 3000 feet for conditions 3 and 4.

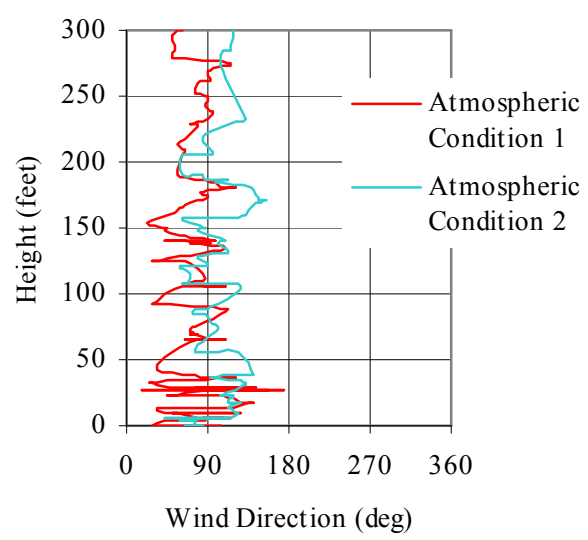

(d). Wind directions for altitudes up to 300 feet for conditions 1 and 2 .

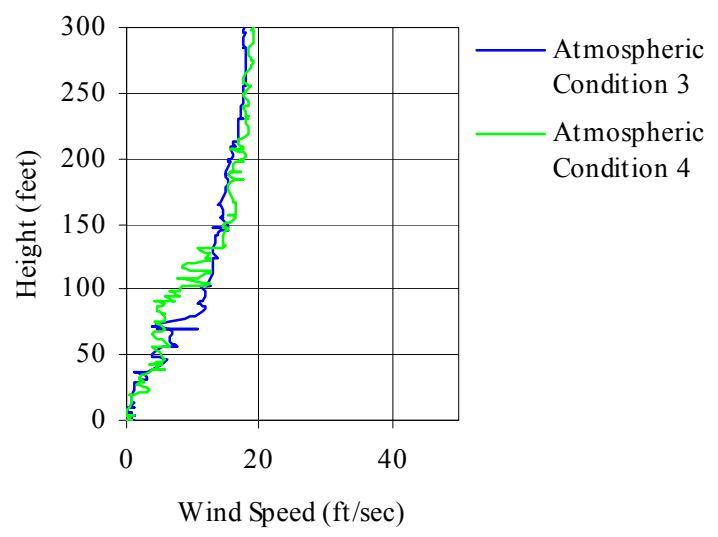

(f). Wind speed profiles for altitudes up to 300 feet for conditions 3 and 4.

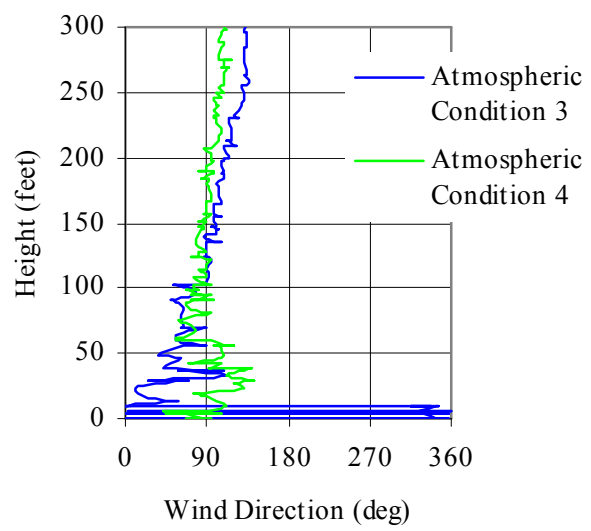

(h). Wind direction for altitudes up to 300 feet for conditions 3 and 4 .

Figure 3. Wind speed and directions for the four atmospheric conditions. 


\section{Acoustic Data.}

The data used to validate the ray tracing code is a subset of the data obtained at Eglin Air Force Base in August and September of 2007. A Mil MI-8 helicopter was used as the acoustic source in all validation cases presented here. Vehicle position, velocity, and heading were recorded for all flights using an onboard GPS system. The vehicle is moving toward the microphone at about $150 \mathrm{kts}$, or $253 \mathrm{ft} / \mathrm{sec}$.

Acoustic data were acquired from a single, ground board mounted, microphone deployed near the east end of the test site as shown in Figure 1. The microphone signal was digitized using a $25 \mathrm{kHz}$ sampling rate, and a low-pass filter at $12.5 \mathrm{kHz}$. The microphone system has a dynamic range of $96 \mathrm{~dB}$ which is sufficient to acquire measurements well below the ambient noise floor, which was between 30 and $35 \mathrm{~dB}$ at the first rotor harmonic of $16.0 \mathrm{~Hz}$ for the test cases shown. At the frequency and distances used for the validation cases, atmospheric absorption is negligible and the propagation loss is dominated by geometric spreading. The sound pressure level of the first main rotor harmonic received by the microphone is shown in Figure 4 for the four validation cases of Table 1. The levels shown here have been adjusted to account for doppler effects.

Case 1

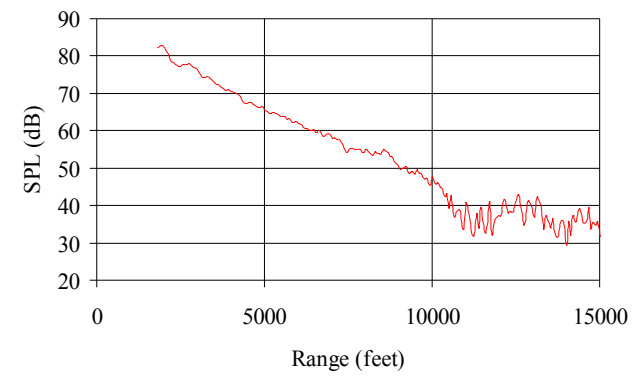

Case 3

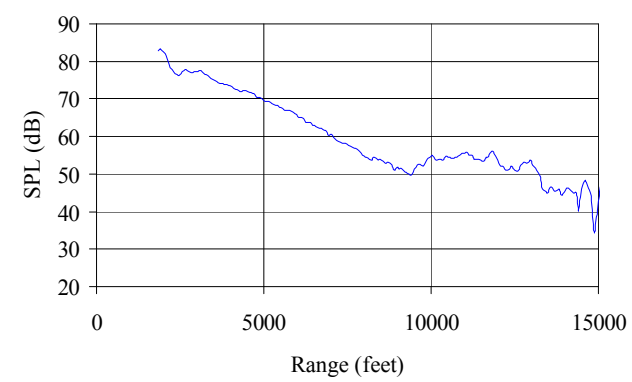

Case 2

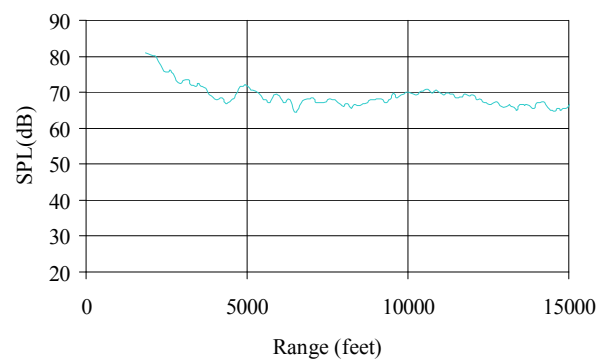

Case 4

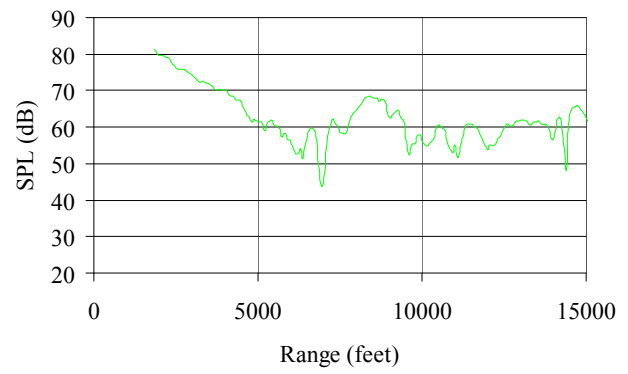

Figure 4. Mil MI-8 measured first main rotor harmonic for each of the four flight cases.

The acoustic source is assumed constant for the purposes of this effort. This assumption is reasonable since rotor noise in steady level forward flight remains relatively constant within $+/-5$ degrees from the plane of the rotor, beyond which the signal amplitude begins to drop rapidly ${ }^{6}$. Since the Mil MI-8 helicopter rotor has a built in pre-tilt of 4.5 degrees from a horizontal plane (see Figure 5), the useable data must be within 9.5 degrees from the horizontal plane. Using the combined 9.5 degrees from the horizontal plane and an altitude of 300 feet, it is assumed that the acoustic source is constant beyond a range of 1792 feet. 


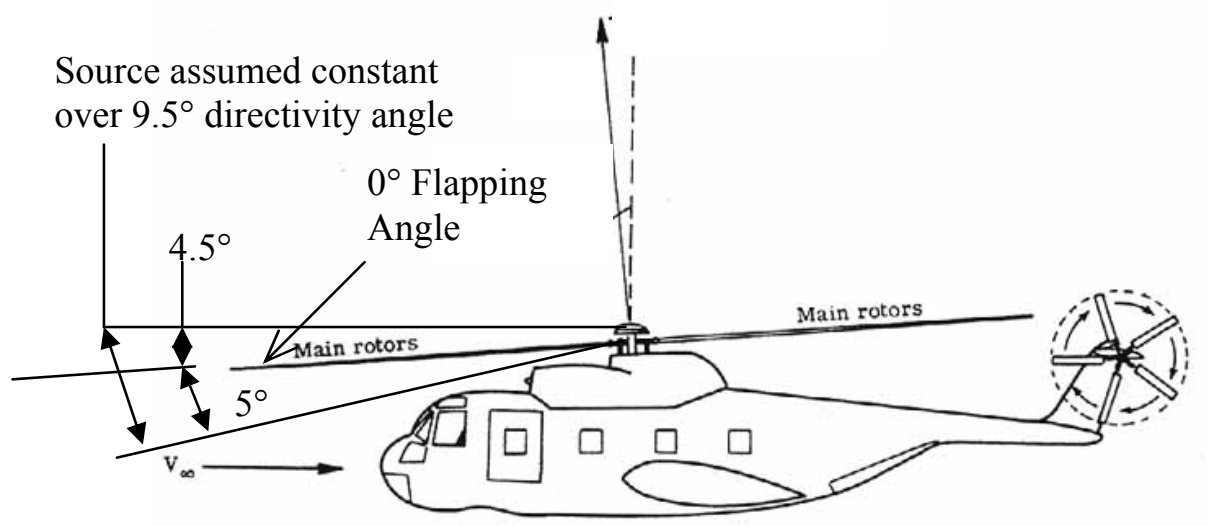

Figure 5. Directivity angle over which the rotor source noise is assumed constant.

\section{Predictions}

Acoustic levels are predicted using the ray tracing propagation code, RTP, for the four atmospheric conditions described above. As stated earlier, the vehicle is moving toward the microphone at approximately the same airspeed and altitude. For the flight test presented, the primary difference between these cases is the relative difference between the direction of the wind and the flight path. The refractive effects are caused by the temperature and wind gradients. It is the intent of this section to describe the refractive effects for forward propagating rays predicted by RTP for these conditions.

RTP predicts sound pressure level (SPL) losses as a function of range, which is defined as the distance along the ground from a position directly under the source (or aircraft). For convenience, the SPL losses are set to zero at a range of zero. The predicted SPL levels are shown in Figures $6 \mathrm{a}$ and $6 \mathrm{~b}$. Currently RTP predictions do not include reflections and therefore stop when the acoustic ray intersects the ground. Points A, B C and D in Figures 6a and 6b correspond to the ground intersection points for the furthest ray that intersects the ground in each case. These rays will be called boundary rays for reference purposes. The figures also show the RTP prediction for a non-refracting constant-atmosphere case.

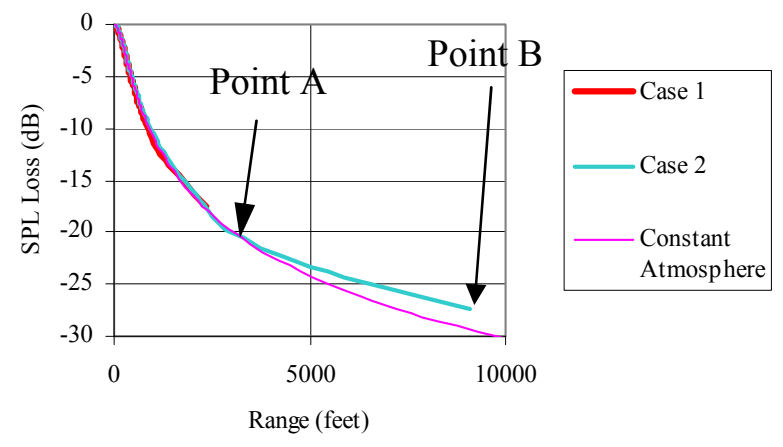

(a). SPL Losses for Case 1 and 2

Figure 6. SPL loss predictions for the four cases.

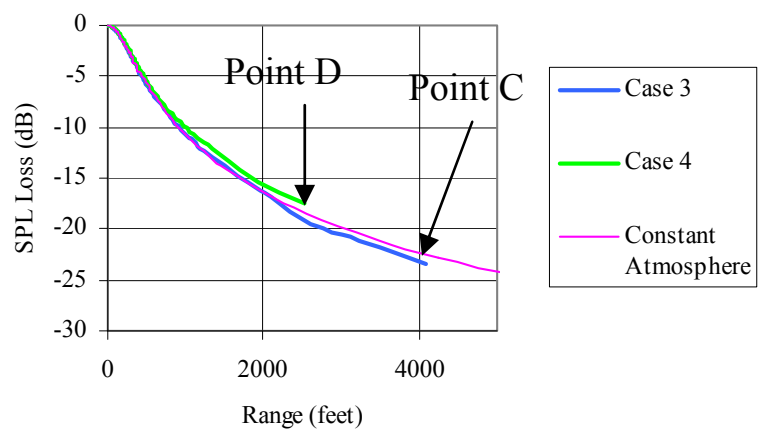

(b). SPL Losses for Cases 3 and 4. 
It is noted that the SPL losses do not differ significantly $(2-3 \mathrm{~dB})$ up to the point at which the rays intersect the ground. This is as expected, since the propagation losses resemble spherical spreading at these short ranges. While RTP does not predict sound levels beyond the boundary ray locations, investigation of the manner in which the rays intersect the ground, along with reasonable assumptions on the refraction effects of the atmosphere, allows one to deduce the acoustic levels in these distant regions. Therefore, it is possible to compare RTP predictions at this stage to ensure the refraction effects are correct before proceeding with reflections and more advanced propagation effects.

Rays are classified as either upward or downward refracting when they intersect the ground. When an upward refracting ray grazes the ground a shadow boundary is formed, and diffraction into the area beyond the shadow boundary results in a shadow region with a rapidly attenuating acoustic signal. Likewise, it is expected that downward refracting rays are reflected by the ground, and will subsequently be refracted back toward the ground by wind and temperature gradients yielding multi-path regions.

It only takes slight changes in atmospheric conditions to dramatically change long range propagation losses. The ability to observe the ray characteristics, and how they change within several atmospheric layers, is important when trying to classify associated regions as either shadow or multi-path beyond the point where the rays intersect the ground. Table 2 summarizes the ray properties for the four cases, including upward or downward refracting, the corresponding expected region's characteristics, and the ray details for both shadow and multi-path regions.

For clarity, an explanation of the ray details in Table 2 is given. For shadow regions, there is one unique ray that will graze the ground for each case, and for obvious reasons it is called the grazing ray. Associated with this grazing ray is a critical elevation angle that describes the angle between the grazing ray and a horizontal plane at the source. Any ray emitted at an angle less than (shallower than) the critical elevation angle will turn before it reaches the ground, and any ray emitted at a larger angle (steeper) will intersect the ground, unless turned by an intermediate layer. The range for both the upward and downward refracting cases is the point where the boundary ray intersects the ground. And finally, the azimuthal extent of the shadow boundary shown in Table 2 is defined by the azimuthal angles in the ground plane (which are referenced with respect to conventional flight path directions) that locate the beginning and the end of the shadow region on the ground. For further explanation of these characteristics see Heath and McAninch ${ }^{5}$.

Table 2. Summary of ray properties for the four cases.

\begin{tabular}{|c|c|c|c|c|}
\hline \multirow{2}{*}{$\begin{array}{l}\text { Ray Properties } \\
\text { Refraction } \\
\text { (upward/downward) }\end{array}$} & Case 1 & Case 2 & Case 3 & Case 4 \\
\hline & upward & downward & undetermined & downward \\
\hline $\begin{array}{l}\text { Region prediction } \\
\text { (shadow/multi-path) }\end{array}$ & shadow & multi-path & undetermined & multi-path \\
\hline \multicolumn{5}{|l|}{$\begin{array}{l}\text { Upward Refracting Ray } \\
\text { Details }\end{array}$} \\
\hline \begin{tabular}{l|l} 
critical elevation \\
angle for grazing \\
ray
\end{tabular} & $11.6^{\circ}$ & NA & $3.7^{\circ}$ & NA \\
\hline $\begin{array}{l}\text { range of shadow } \\
\text { boundary }\end{array}$ & $2355 \mathrm{ft}$ & NA & $4079 \mathrm{ft}^{*}$ & NA \\
\hline \begin{tabular}{|l|} 
azimuthal extent \\
of shadow region \\
(with respect to \\
flight path \\
direction)
\end{tabular} & $\begin{array}{l}10^{\circ}-200^{\circ} \\
\left(\Delta 190^{\circ}\right)\end{array}$ & $\begin{array}{l}30^{\circ}-220^{\circ} \\
\left(\Delta 190^{\circ}\right)\end{array}$ & $\begin{array}{l}80^{\circ}-170^{\circ} \\
\left(\Delta 90^{\circ}\right)\end{array}$ & $\begin{array}{l}40^{\circ}-200^{\circ} \\
\left(\Delta 160^{\circ}\right)\end{array}$ \\
\hline \multicolumn{5}{|l|}{$\begin{array}{l}\text { Downward Refracting } \\
\text { Ray Details }\end{array}$} \\
\hline \begin{tabular}{l|l} 
range of boundary \\
ray
\end{tabular} & NA & $9075 \mathrm{ft} * *$ & NA & $2516 \mathrm{ft}$ \\
\hline \multicolumn{5}{|c|}{$\begin{array}{l}\text { * The upward refracting ray that has a critical elevation angle of } 3.7 \text { degrees turns before it reaches the } \\
\text { ground. The boundary is defined by a downward refracting ray at an elevation angle of } 5.4 \text { degrees. } \\
\text { Rays below an elevation angle of } 0.7 \text { degrees turn before they reach the ground. The boundary ray definec } \\
\text { here, with a range of } 9075 \mathrm{ft} \text {, is the ray defined by an elevation angle of } 0.7 \text { degrees. }\end{array}$} \\
\hline
\end{tabular}


Ray paths have been traced from the source to the ground for several elevation angles and are shown in Figures 7 and 8 for cases 1 through 4 . Each plot shows several downward propagating rays evenly spaced at 0.1 degree increments from either the horizontal source plane for downward refracting rays, or from the critical elevation angle for upward refracting rays. The boundary rays for cases 1 through 4 intersect the ground at points $\mathrm{A}, \mathrm{B}, \mathrm{C}$ and $\mathrm{D}$ at ranges between 2000 and 10000 feet as shown in Table 2. These points correspond with those shown in Figures $6 \mathrm{a}$ and $6 \mathrm{~b}$ above.

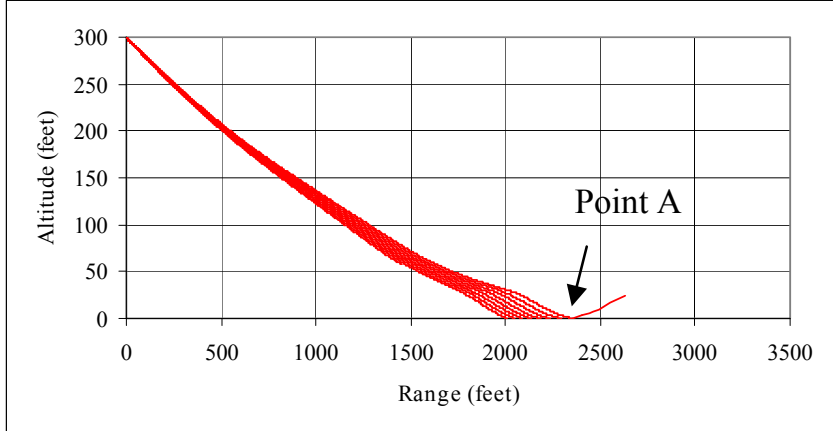

(a). Case 1. Traced ray paths at elevation angles of 11.7 through 13.7 in increments of 0.1 degrees. The critical emission angle is 11.6 degrees and the boundary ray grazes the ground at a range of 2355 feet (point A).

Figure 7. Traced ray paths for case 1 and case 2 .

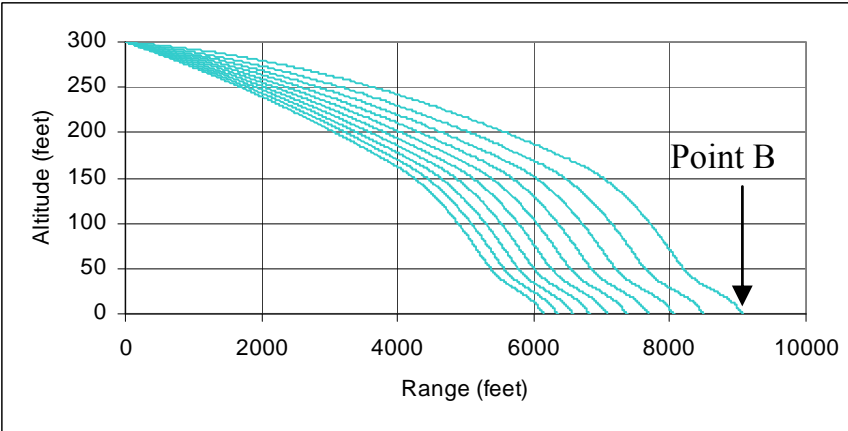

(b). Case 2. Traced ray paths at elevation angles of 0.7 through 1.5 degrees in increments of 0.1 degrees. The boundary ray intersects the ground at a range of 9075 feet (point B).

Case 1 prediction: Case 1 conditions indicate the formation of a shadow region. The shadow boundary is well defined, at 2355 feet, and the adjacent rays behave similarly, as shown in Figure 7a.

Case 2 prediction: Case 2 conditions indicate the formation of a multi-path region based on the fact that the rays are clearly downward refracting as shown in Figure 7b. The rays remain downward refracting and will produce a multipath region beyond the boundary ray intersection range of 9075 feet.

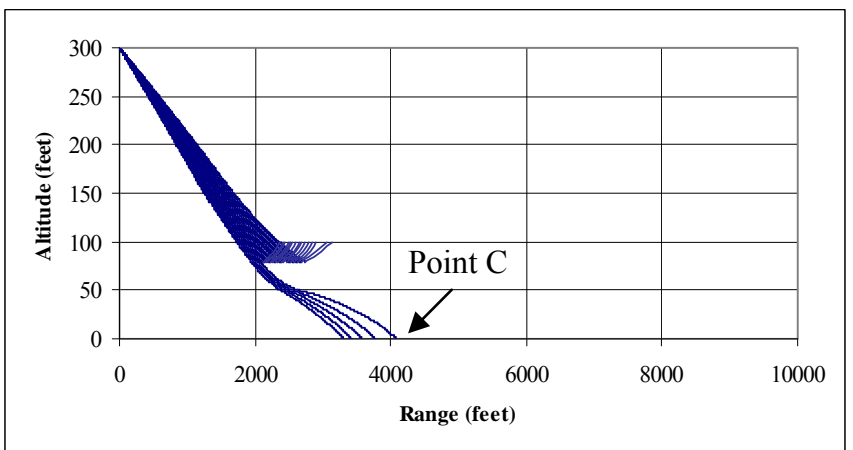

(a). Case 3. Traced ray paths at elevation angles of 3.8 through 5.8 degrees in increments of 0.1 degrees. The critical emission angle is 3.7 degrees and the first ray that intersects the ground is at a range of 4079 feet (point C).

Figure 8. Traced ray paths for case 3 and case 4 .

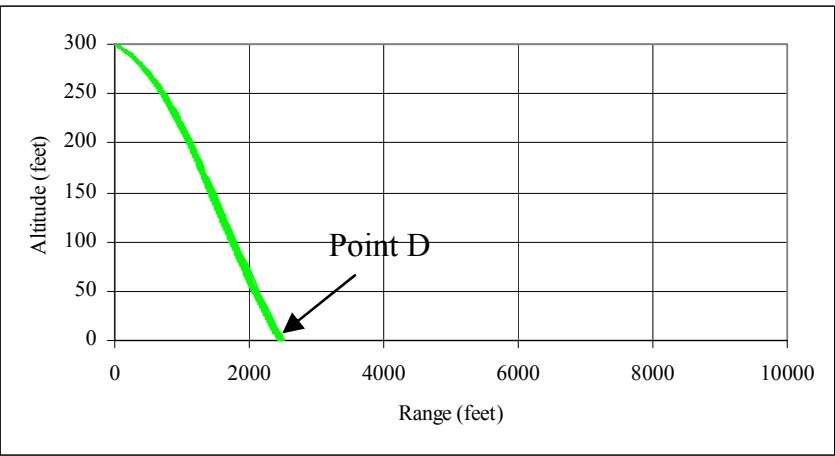

(b). Case 4. Traced ray paths at elevation angles of 0.1 through 2.7 degrees in increments of .1 degrees are shown. The boundary ray intersects the ground at a range of 2516 feet (point $\mathrm{D}$ ).

Case $\underline{3}$ predictions: Case 3 conditions indicate neither a shadow region, nor a multi-path region. It can be seen in Figure 8a that the first fifteen rays are upward refracting, but turn away from the ground in an intermediate atmospheric layer between 50 and 75 feet. The adjacent rays are downward refracting and intersect the ground at ranges of 4079 feet and less. Since this case is anything but "ideal", one might expect the downstream region to be affected by both the upward and downward refracting rays. 
Case 4 predictions: Case 4 conditions indicate the formation of a multi-path region. The rays shown in Figure $8 \mathrm{~b}$ are downward refracting and very compact, in fact, there are 27 rays (covering the first 2.8 degrees of elevation). In addition, it should be noted that this case lies along a flight path angle of 20 degrees which is near the asymptote where downward refracting rays change to upward refracting rays, which occurs at an azimuthal angle of 40 degrees as stated in Table 2.

Both shadow regions and multi-path regions have distinct characteristics. Acoustic levels in the shadow region would be expected to drop instantly to zero if it were not for diffracted sound leaking into the region. The acoustic pressure in the diffracted region decays exponentially ${ }^{7}$ as $\mathrm{p} \propto \mathrm{e}^{-\alpha \Delta \mathrm{r}}$, where $\Delta \mathrm{r}$ is the distance from the shadow boundary, and $\alpha$ is a coefficient based on the temperature and wind gradients, the ground impedance, source frequency, and curvature of the grazing ray. This exponential decay becomes a straight line when plotting SPL versus range as shown in Figure 9. A coefficient of $.0004 / \mathrm{ft}$ was used as the diffraction estimate for the two diffraction cases (cases 1 and 3). The diffraction is assumed to begin around 2500 feet.

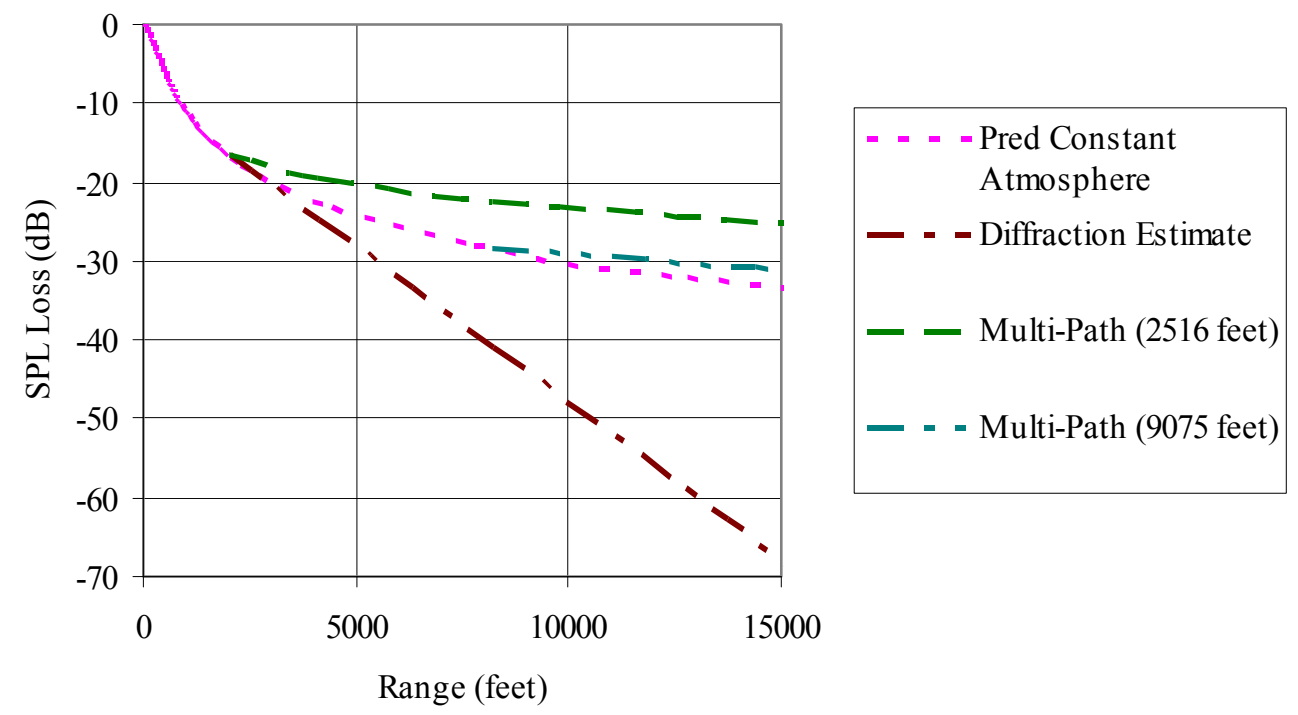

Figure 9. Extended predictions for shadow and multi-path regions.

Multi-path regions on the other hand are dominated by multiple reflections from multiple rays and acoustic pressure decays as $1 / \sqrt{\mathrm{r}}$, or equivalently an SPL loss of $3 \mathrm{~dB}$ per range doubling. This loss is shown in Figure 9 for the two conditions, one beginning at a range of 2516 feet to correspond with point $\mathrm{D}$ (case 4), and a second beginning at 9075 feet to correspond with point B (case 2). SPL losses for a non-refracting constant-atmosphere case at the same source altitude of 300 feet are also shown.

\section{Comparison of Measured and Predicted}

Sound pressure level losses for the $16 \mathrm{~Hz}$ main rotor first harmonic are shown in Figure 10 for cases 1 and 2, and in Figure 11 for cases 3 and 4. Comparison of the two figures (which have similar flight paths for cases 1 and 3, and cases 2 and 4, see Table 1) shows large differences in observed losses for moderate changes in the weather conditions. For example, a SPL difference of $24 \mathrm{~dB}$ is seen between the shadow region and the multi-path region for cases 1 (flight path direction of 100 degrees) and 2 (flight path direction of 20 degrees) for the $1^{\text {st }}$ rotor harmonic at a range of 10,000 feet. On the contrary, a difference between cases 3 (flight path direction of 100 degrees) and 4 (flight path direction of 20 degrees) is sporadic and shows only a $3 \mathrm{~dB}$ SPL difference at 10,000 feet. These differences, at least in part can be explained by comparing the ray details predicted by RTP.

Figure 10 compares the SPL losses for predictions and measurements as a function of range. Since the absolute acoustic source strength of the helicopter is unknown, comparisons between the predicted and measured SPL losses 
are achieved by normalizing the data at a range of $2500 \mathrm{ft}$. This is valid if the noise source is assumed to be constant for the duration of each flight. The predictions are shown as "Predicted Case 1" and "Predicted Case 2" for the short ranges, and as "Predicted Diffraction" and "Predicted Multipath (9075 feet)" for the long ranges. Figure 10 also contains the non-refracted prediction labeled "Predicted Constant Atmosphere" and the ambient noise floor. The measurements for case 1 and case 2 show definite shadow and multi-path regions resulting from refraction.
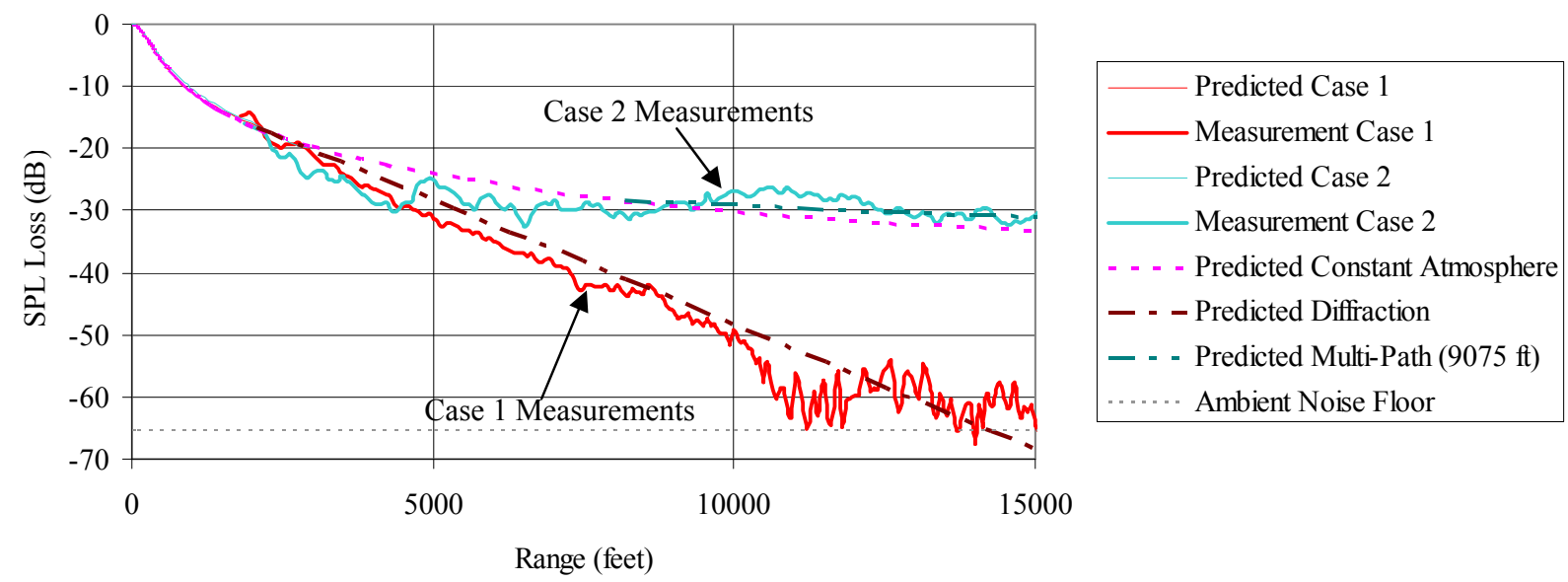

Figure 10. Measured vs Predicted SPL losses for Cases 1 and 2.
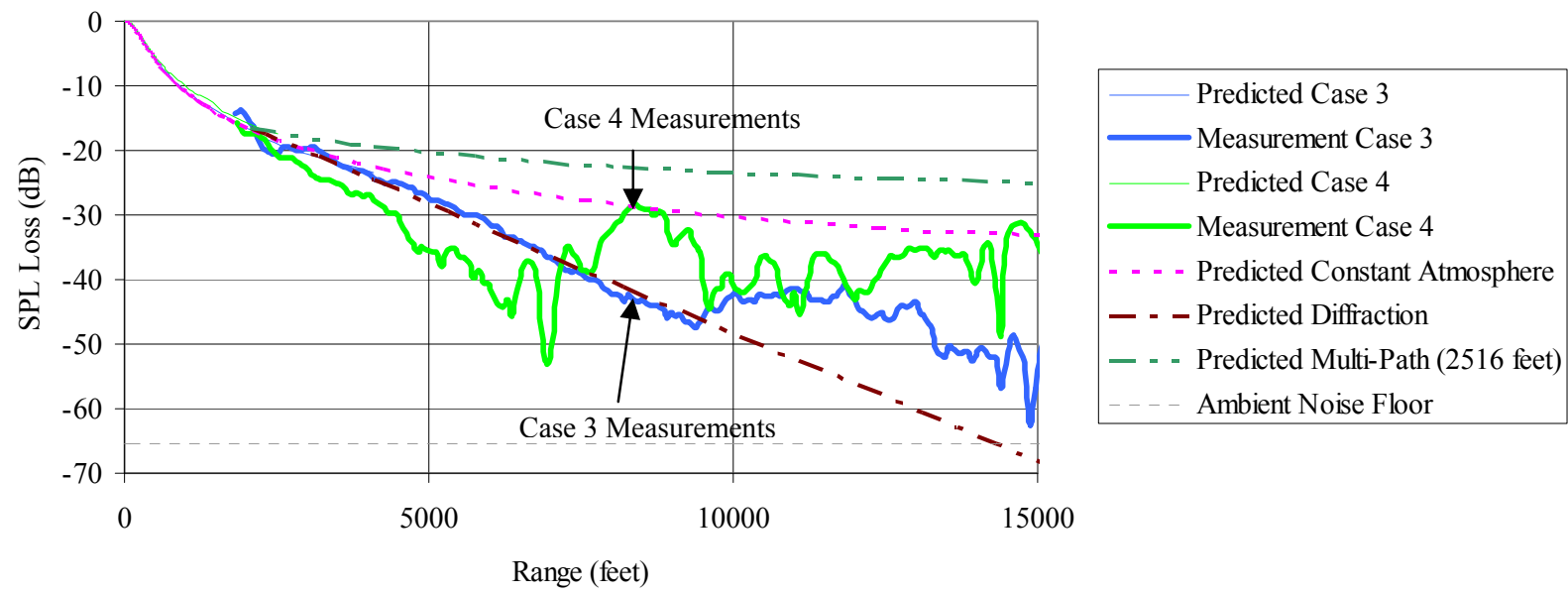

Figure 11. Measured vs Predicted SPL losses for Cases 3 and 4.

Case 1: The SPL loss measured for case 1 in Figure 10 has a clear and steep decline as predicted, until the signal falls to the ambient noise floor, which confirms the presence of a shadow region. The SPL decay within the shadow region also begins near the predicted shadow boundary range of 2355 feet and is consistent with the diffraction predictions described earlier.

Case 2: Case 2 measurements are shown in Figure 10. An initial SPL loss is seen between 2000 and 4000 feet. This decline is steeper than expected for a multi-path region, but by 5000 feet the levels are consistent with multipath characteristics. It should also be noted that for these particular conditions there is little difference between the constant atmosphere and multi-path predictions.

Figure 11 compares predicted cases 3 and 4 to the measurements. Like Figure 10, SPL loss measurements are shown as a function of range. The predictions are labeled as "Predicted Case 3" and "Predicted Case 4" for the short 
ranges, and as "Predicted Diffraction" and "Predicted Multipath (2516 feet)" for the long ranges. The figure also contains the constant atmosphere prediction and the ambient noise floor. The measurements do not follow either the ideal shadow or the multipath regions for these cases.

Case 3: The data for case 3, as seen in Figure 11, show a well defined shadow region in the beginning, but the shadow region becomes overpowered by a multi-path region just beyond 10,000 ft. Since the predicted rays can neither define a shadow nor a multi-path region, it is not surprising to see a mixture of the two effects.

Case 4: Case 4 does not act like a multi-path region as originally predicted, but instead has a very steep drop in the sound pressure levels before it switches to a multi-path region at about 6000 feet (see Figure 11). A definite cause for this behavior is unknown, however it is suspected that either the flight path heading may be located along the transition zone between downward and upward refraction, or, possibly that the multipath region occurs beyond the ranges shown here.

The physical effects predicted by RTP are seen in the flight data, with the exception of case 4 . The comparisons between Eglin test data and predictions, where well defined refractive properties exist, are consistent. The location of shadow region formation and the regions of multi-path propagation show reasonable agreement. The rapid attenuation observed within the shadow region is accurately predicted using established diffraction theory.

\section{Summary}

Validation of NASA's current propagation capabilities using the ray-tracing code, RTP, is an important step toward developing better prediction methods.

Incorporating wind and temperature refractive effects into the propagation capabilities was the first of several key steps in predicting the measured sound pressure levels seen during the 2007 Eglin Rotorcraft Test. Numerical predictions from RTP are presented and agree with measurements for three of the four flight test cases. RTP is able to predict most of the sound pressure level variations seen during the flight tests, and thus, serves to partially validate the modeled wind and temperature refractive effects.

\section{References}

${ }^{1}$ Heath, S.L., "RTP- Ray Tracing Propagation Code User's Manual," NASA Technical Memorandum, National Aeronautics and Space Administration, Langley Research Center, Hampton, VA, in progress.

${ }^{2}$ Blokhintsev, D. I., “Acoustics of a Nonhomogeneous Moving Medium,” NACA TM-1399, 1946.

${ }^{3}$ Lighthill, M. J., "The Fourth Annual Fairey Lecture: The Propagation of Sound Through Moving Fluids," Journal of Sound and Vibration, Vol. 24(4), Oct. 1972, pp. 471-492.

${ }^{4}$ Lewis, R.M. and Keller, J. B., "Asymptotic Methods for Partial Differential Equations: The Reduced Wave Equation and Maxwell's Equations”, New York University Research Report No. EM-194, January 1964.

${ }^{5}$ Heath, S. L., and McAninch, G. L., "Propagation Effects of Wind and Temperature on Acoustic Ground Contour Levels," AIAA Paper 2006-0411, 2006.

${ }^{6}$ Hubbard, Harvey H. Hubbard (Ed.), “Aeroacoustics of Flight Vehicles: Theory and Practice, Chapter 2 - Rotor Noise by Schmitz, F. H.," NASA Reference Publication 1258, Vol.1, National Aeronautics and Space Administration, Langley Research Center, Hampton, VA, August 1991.

${ }^{7}$ Pierce, A.D., Acoustics: An Introduction to Its Physical Principles and Applications, $1^{\text {st }}$ ed., McGraw-Hill, New York, 1981. 